Cuestiones de filosofía

ISSN: 0123-5095

Vol. 1 - No. 17

Año 2015

pp. $104-117$

\title{
DE ESTELAS Y ESCOMBROS: ENSAYO SOBRE LA OBRA DE NICOLÁS GÓMEZ DÁVILA
}

\author{
Of Trails and Debris: \\ Essay on the Work of Nicolás Gómez Dávila
}

\author{
Carlos Andrés Ulloa-Rivero* \\ Zatara1987@gmail.com \\ Universidad la Gran Clombia (Colombia)
}

Fecha de recepción: 10/11/2014

Fecha de evaluación: 13/03/2015

Fecha de aprobación: 13/09/2015

\begin{abstract}
"Quien dice la verdad, casi no dice nada"
Antonio Porchia-Voces
\end{abstract}

\section{Resumen}

Este ensayo reflexiona sobre la obra de Nicolás Gómez Dávila, desde dos momentos: el primero, en su relación con el pensamiento colombiano y su pertenencia a él, y el segundo, a partir de las condiciones propias que esta obra exige a las investigaciones que profundizan sobre ella. Contrario a la idea que ubica la obra de este autor fuera de cualquier categoría del pensamiento colombiano, el primer momento de este ensayo elabora tres argumentos por los cuales dicha creencia puede ser reinterpretada desde una nueva perspectiva, que no desconoce, sin embargo, su vinculación, en principio problemática, con las líneas del pensamiento colombiano. Posteriormente,

* Estudiante del programa de Doctorado en Filosofía de la Universidad Nacional de Colombia. Investigador Grupo de Investigación: Estudios en Educación, Pedagogía y Nuevas Tecnologías. Categoría D, Colciencias, 2013/2014. 
una visión de conjunto de la investigación que hasta el momento se ha realizado en torno a este autor pondrá en evidencia una dificultad que debe ser entendida como una condición propia de esta obra filosófica. Dicha condición es presentada, pues, como un aspecto que ha de ser asumido por todas las investigaciones futuras que quieran profundizar en el pensamiento de este autor, y revelar así todo su potencial filosófico.

Palabras clave: Nicolás Gómez Dávila, Filosofía colombiana, Pensamiento colombiano del siglo XX.

\begin{abstract}
This paper considers two aspects of the Colombian philosopher Nicolas Gómez Dávila's work. The first one concerning the place of this philosopher viewed from the tradition of the different Colombian schools of thought. The second one is focus on analyzing the different aspects of his work, that will become afterwards a set of methodological requirement for any future research on it. Contrary with the idea that usually places this philosopher out of any Colombian school of thought, in the first moment of this paper, we will present three arguments by which we think this old conception must be revised, even if our hypothesis do not neglect the fact that any belonging of this philosopher to the tradition of Colombian philosophy is highly problematic. Subsequently, after a revision of the research that has been made about this thinker, we will exhibit that common difficulty on it, which must be understood as a specific condition of this particular philosophical work. Therefore, this condition is presented as an unavoidable aspect that any future research should assume in order to go deeply in the philosophical thought of Nicolás Gómez Dávila.
\end{abstract}

Keywords: Nicolás Gómez Dávila, Colombian Philosophy, 20th Century Colombian Thought. 


\section{Nicolás Gómez Dávila y el Pensamiento en Colombia}

Comencemos afirmando que la obra de Nicolás Gómez Dávila constituye un paso obligado en cualquier estudio o antología que busque retratar el pensamiento colombiano del siglo XX. Es claro, no obstante, que esta afirmación es problemática y debe,en primer lugar, hacer frente a ciertas objeciones, una de las cuales se encuentra ya señalada por Alfredo Abad Torres, en uno de los libros más completos dedicados a nuestro autor: Pensar lo implícito en torno a Gómez Dávila (2008). Allí se expone la imposibilidad de trazar una continuidad intelectual entre las corrientes de pensamiento filosófico colombiano -por ejemplo aquellas que marcaron el siglo XIX-ylascualidades atípicas de este pensador, denominado, comúnmente, solitario. Lo anterior bajo la idea de que un breve vistazo a la obra gomezdaviliana bastaría para revelarnos que ni el utilitarismo, ni el positivismo, ni el liberalismo clásico son fuentes de influencia histórica en su pensamiento ni representaron para el bogotano algún tipo de interés más allá del de la descalificación ${ }^{1}$.

Tampoco es clara la relación, que en tantos otros intelectuales del país sí lo fue, con las corrientes filosóficas que marcaron del siglo XX colombiano, a saber, la fenomenología y el marxismo ${ }^{2}$. Estas, pese a que se trata de corrientes rastreables dentro de su obra, no recibieron el mismo tratamiento de quienes se las apropiaron y las debatieron en el espacio público de la discusión intelectual, como en el caso, por ejemplo, de Danilo Cruz Vélez o de Estanislao Zuleta. Contrario a lo anterior, el uso que Gómez Dávila hizo de estos desarrollos del pensamiento occidental está profundamente ligado a la fuerza de sus convicciones, resultando así que, en sus obras, todo diálogo intelectual con las corrientes filosóficas - tanto las que impactaron su época como las que no- no se expone de forma explícita, sino implícita y parcial. Subyace a esta actitud una fuerte convicción sobre la libertad del pensamiento, sobre el quehacer libre de la inteligencia, y un recelo profundo que contrapone a estas altivas creencias una visión completamente peyorativa del ejercicio académico profesionalizado.

1 "Éticas utilitaristas o sociales, estéticas naturalistas o expresionistas, epistemologías pragmáticas o instrumentales, intentan reducir el valor a su esquema prepuesto, y no son más que artefactos ideológicos" (Gómez Dávila, 2002, p. 69). Todas las citaciones provenientes de la obra de Nicolás Gómez Dávila provienen de sus reediciones por Villegas Editores.

2 Seguimos en este punto el análisis que Guillermo Hoyos Vásquez realiza en su artículo: "Medio Siglo de Filosofía Moderna en Colombia. Reflexiones de un participante". Revista de Estudios Culturales(Bogotá), 1999, N. ${ }^{\circ}$ 03, junio. pp. 43-58. 
Partiendo del hecho, por lo tanto, de que parece imposible subsanar la deliberada ausencia de tan singular pensador del espacio colombiano de discusión académica, hay quienes han intentado, por otra parte, ensalzar su papel histórico en el país presentándolo como un eximio representante de una de nuestras fuerzas políticas hegemónicas: el Partido Conservador colombiano. Sin embargo, este intento -quizás más emocional que intelectual-pareciera también estar condenado a fracasar, pues una atenta revisión a las bases históricas de este movimiento político, como sucede en el trabajo de Michaël Rabier (2014), claramente establece la diferencia entre el conservadurismo y la actitud reaccionaria, que Gómez Dávila no solo estudió, sino que intentó encarnar durante su vida. Análisis de este tipo nos muestra, pues, que la figura de Gómez Dávila ostenta una serie de particularidades que dificultan cualquier asociación apresurada a su contexto.

Sin embargo, pese a que la suma de estas particularidades sea entendida como una serie de obstáculos infranqueables, pese a la cualidad de universal que los principales divulgadores de su obra reconocen en ella (véase, Volpi, 2005,) y, aún más, pese al declarado menosprecio y desconfianza que este autor sintió con respecto a sus compatriotas ${ }^{3}$, existen aún tres argumentos que nos permitirían asumir esta situación desde una nueva perspectiva y sustentar asíel justo lugar que Nicolás Gómez Dávila ha de tener dentro de la historia del pensamiento colombiano.

El primer argumento, que ya se encuentra desarrollado claramente en el artículo de Guillermo Hoyos: "Don Nicolás Gómez Dávila, pensador en español y reaccionario auténtico" (2008), consiste en destacar, justamente, que, anterior a la universalidad de sus ideas, Gómez Dávila ostenta la cualidad ineludible de ser un pensador en español, una cualidad que inmediatamente lo referencia con la comunidad lingüística que comparte. Es un dato conocido que el diálogo que el bogotano estableció con la cultura de Occidente, a través de su exuberante biblioteca, fue llevado a cabo

3 Constatan dicha actitud los escasos escolios que este autor dedica a Suramérica, Colombia y su población:"Características del colombiano: imposibilidad de lo concreto; en sus manos todo se vuelve vago; falta de moralidad; la noción del deber le es desconocida; la única regla es el miedo del gendarme o del diablo; en su alma ninguna estructura moral, ni intelectual, ni social; ignora toda tradición; sometido pasivamente a cualquier influencia, nada lo marca; nada fructifica, ni dura en ese suelo de contextura informe, movedizo, plástico e inconsistente" (2003, p. 153);"Cuando se presenta la ocasión de hacer alguna bajeza, el colombiano rara vez la desperdicia" (2003, p. 432); "No ser un profesional de la literatura me procura el eximio privilegio de eximirme de la obligación de leer los libros de mis compatriotas" (2003, p. 420). 
respetando las lenguas originales de sus autores, tanto así que él mismo afirmaba sobre la relación entre lenguaje y filosofía lo siguiente:

Toda filosofía está pensada en la sustancia misma de un idioma; se engendra en una materia verbal. Traducir una filosofía es cosa imposible, ya que destruimos su sentido al suprimir el orden lingüístico al que pertenece y al que se refieren para alcanzar su pleno valor los conceptos más abstractos (2003, p. 69).

Luego la pregunta no se hace esperar, y es la siguiente: ¿por qué no asumir esta misma actitud frente a la obra gomezdaviliana y asumir, entonces, la responsabilidad que tenemos, como hispanohablantes, de hacer frente a la enorme tarea de estudio y de trabajo que nos plantea, en lugar de esperar pasivamente hasta que autoridades académicas internacionales sean las que promuevan su valor filosófico? Desde esa perspectiva, la necesidad de incluir la voz de este colombiano dentro de los espacios académicos latinoamericanos se hace no solo evidente, sino propicia.

El segundo argumento es la constatación de la influencia histórica que Gómez Dávila ejerció sobre algunas de las figuras intelectuales más sobresalientes del país a mediados del siglo $\mathrm{XX}^{4}$. Esta influencia, que hasta el momento no ha sido objeto directo de estudio, salvo en contadas excepciones ${ }^{5}$, se ha limitado comúnmente al señalamiento anecdótico de las tertulias que con cierto grupo de amigos íntimos compartía los domingos en la tarde en la casa del bogotano; sin embargo, así se limitara a denotar la enorme admiración y el profundo respeto que este pensador causó en quienes compartieron con él, el hecho escueto de que tantos y tan variados personajes de la

4 El documento que hasta el momento mejor expresa esa influencia puede ser aquel en la que sus contertulios y amigos escriben en ocasión al fallecimiento de quien, en varias ocasiones, fue reconocido como su mentor. Aludimos aquí la conocida edición homenaje a Nicolás Gómez Dávila, de la Revista del Colegio Mayor de Nuestra Señora del Rosario(Bogotá), LXXXI, 1988, N. ${ }^{\circ} 542$ (abril-junio), en donde escriben: Alberto Zalamea, Francisco Pizano de Brigard, Hernando Téllez, Álvaro Mutis, Juan Gustavo Cobo Borda. A su vez, reenviamos al lector a la semblanza que sirve de prólogo a Escolios a un texto implícito. Selección, Bogotá, Villegas Editores, 2011, a cargo Mario Laserna Pinzón.

5 Dichas excepciones se empiezan a fraguar, por ejemplo, desde estudios que buscan analizar la influencia literaria de Gómez Dávila en la divulgación de su obra realizada por la revista Eco, trabajo que preparan Nicolás Felipe Díaz, Tomás Molina, Nicolás Antonio Barguil, habiendo este último presentado, además, una ponencia en el Congreso Internacional Nicolás Gómez Dávila en su centenario, sobre la lectura de Ernesto Volkening de los Escolios, basándose en los cuadernos que de este último se encuentran ahora en la Colección Nicolás Gómez Dávila, de la Biblioteca Luis Ángel Arango. 
vida intelectual del país repararan en su obra y coincidieran en exaltar lo extraordinario de su valor es un argumento suficiente para que sea reconocida como un momento importantísimo dentro del paisaje intelectual colombiano del siglo precedente.

Finalmente, el tercer argumento recae sobre la importancia de evaluar los criterios bajo los cuales un autor o una obra pasan a convertirse en patrimonio de un país. Sin descartar el hecho de que cualquier filosofía tiene una relación real con un contexto y una tradición (abandonando así el mito de una reflexión solitaria y universal), y reconociendo, pues, que Gómez Dávila ubicó su diálogo con la tradición filosófica eminentemente europea, lejos así de su presente inmediato o de las problemáticas que allíse vivían, no podemos pretender que su presencia siga pasando inadvertida en el panorama nacional, y sobre todo cuando el momento actual nos reitera que esto no es así. En la actualidad, el panorama ya no coincide con aquel que Álvaro Mutis describía en los años ochenta sobre la obra gomezdaviliana, que apenas comenzaba a ver la luz después de haber sido "celosamente conservada en la penumbra". Hoy, un año después de haberse celebrado públicamente el centenario de su natalicio, a través de varios homenajes y congresos; cuando contamos con la publicación de una reedición completa de sus obras, y podemos constatar el creciente interés e importancia que su pensamiento ha comenzado a reclamar en la comunidad académica, por la multiplicación de trabajos tanto de estudio como de divulgación que versan en su nombre, claramente podemos concluir que la presencia de su pensamiento es un hecho que no puede seguir pasando inadvertido.

Este tercer argumento supone, pues, la necesidad imperiosa de abandonar posturas irreflexivas a la hora de analizar la historia intelectual nacional, pues podrían conducirnos, por ejemplo, a renegar sin más argumentos de quien, primero, quiso renegar de su país, o bien, a ensalzar exageradamente una figura sobresaliente por motivos más chovinistas que intelectuales. Lejos de todo esto, nuestra necesidad es lograr asumir una forma más neutral y objetiva en la tarea crítica e histórica que busca rastrear las fuentes de pensamiento que han sido y que son capaces, aún hoy, de ejercer una "notable influencia en las opiniones del país", recordando con ello las intenciones que animaron el trabajo histórico de Jaramillo Uribe, en El pensamiento Colombiano del siglo XIX (1964).

Desde esta perspectiva, fomentar el trabajo académico sobre la obra de Gómez Dávila no solo resulta coherente, dada su creciente popularidad e influencia, sino beneficioso, por cuanto el espacio de discusión académica no puede sino enriquecerse a través del esfuerzo que supone vincular e incluirlas obras filosóficas 
que, por su estructura e intención, nos exigen repensar los criterios metodológicos específicos que deben imperiosamente ser tenidos en cuenta si se quiere extraer lo mejor de ellas. En lo que resta del ensayo intentaré, pues, dar cuenta de algunas de las condiciones particulares de la obra filosófica de Nicolás Gómez Dávila, teniendo como referente un ideal de investigaciones futuras.

\section{CONDICIONES DE UNA OBRA FILOSÓFICA SINGULAR}

La evidencia de la enorme riqueza intelectual que ostenta la obra gomezdaviliana se ha comenzado a vivir como una invitación que la comunidad académica colombiana está respondiendo activamente. Quien hasta entonces era un "ilustre desconocido", para repetir el epítome con que José Miguel Oviedo lo nombró en su Breve historia del ensayo hispanoamericano (1991); quien hasta el momento era tenido por un amante de las bibliotecas, y cuya obra intelectual, en su momento, no fue más que una rareza dentro de las bibliotecas ajenas se ha convertido hoy en un tema cada vez más activo de investigación. Dicha investigación, que no haríamos mal en concebirla en un momento primario de reconocimiento y divulgación, yque podríamos datar del año 2000 en adelante, es toda aquella que se ubica más allá de las primeras y contadas manifestaciones literarias que generó la obra del bogotano. De este primer momento de la investigación podemos vislumbrar un tema constante de reflexión que ha sido la forma particular de la obra gomezdaviliana: su escritura corta y a manera de glosa, casi colindando con los aforismos (véase para ello: Volpi, 2005; Abad Torres, 2008; Mejía Mosquera, 2007). Al mismo tiempo, las reflexiones en torno a la política y, en especial, al pensamiento reaccionario han sido otro eje central de dicha investigación (véase: Rabier, 2013, Hoyos, 2008, Serrano, 2011).

Ahora bien, a riesgo de que esta valoración pueda sonar injusta opretenciosa, no parece descabellado afirmar que es posible reconocer una falencia común dentro de este primer momento de la investigación, entendiendo dicha falencia como una limitación en la profundidad de los estudios que, precisamente, intentan hacerle frente a una obra que sobresale por su hondura intelectual. La dificultad detrás de esta limitación solía explicarse en ocasiones por el carácter fragmentario de la obra, el cual ocasiona que esta corra el riesgo de ser aprehendida únicamente a través de fáciles manipulaciones. Empero, actualmente nuevas reflexiones están tratando de dilucidar a fondo las dificultades que plantea la obra gomezdaviliana, encontrando así que estas, al parecer, van más allá de su presentación dispersa y asistemática, y recaen, en cambio, sobre la misma voluntad creativa de su autor, que, en consonancia 
con la fórmula de Leo Strauss, que Michaël Rabier actualiza, para el pensamiento del colombiano, habría decidido escribir sus obras desde una "estrategia esotérica". Escribir bajo una estrategia esotérica supone hacer un uso reservado de las referencias intertextuales de una obra, logrando con ello determinar de antemano quiénes habrán de ser sus lectores ideales, a saber: aquellos privilegiados que puedan responder al llamado silencioso que murmuran las referencias implícitas del texto ${ }^{6}$.

No son pocos los escolios que el propio Gómez Dávila destina para dejar en claro los principios de escritura desde los cuales estima su obra, uno de los cuales afirma, por ejemplo,que: "El escritor bien educado trata de ser claro. Pero no achaquemos siempre nuestra ineptitud a su mala educación. Explicar, en vez de aludir, supone desprecio al lector" (2005a, t. I, p. 40). Este punto, que fácilmente podría ser identificado como un recelo aristocrático, es, no obstante, la evidencia de una convicción más profunda acerca de lo que Gómez Dávila considera que es el ejercicio propio de la inteligencia y su relación con la verdad: convicción que ha de imponerse luego como condición de estudio a la obra gomezdaviliana.

La escogencia de la escritura corta, reducida a la glosa y el comentario, puede entenderse bajo la forma como Gómez Dávila quiere expresar una visión sobre la inteligencia humana, la cual, más que representar una capacidad de aproximación estática y contemplativa de la realidad, se vive para el autor como una extensión de la sensibilidad que, al igual que ese impulso vital que mueve al organismo a ir más allá de sí mismo, corresponde a ese principio dinámico por el cual el hombre desea y ambiciona el sentido de las cosas. Este principio de una inteligencia dinámica y en continuidad con la sensibilidad del hombre se soporta en Gómez Dávila bajo la convicción de que la verdad no puede ser una mera abstracción ni una adecuación

6 El sobresaliente trabajo Michaël Rabier no solo ha estado orientado a trazar las líneas del pensamiento político de Gómez Dávila, sino que también ha versado en trabajo investigativo sobre el enorme patrimonio intelectual, ahora público, al haber sido adquirido por la biblioteca Luis Ángel Arango,que componía la biblioteca del bogotano. Es justamente a partir del reconocimiento de este espacio desde el cual se engendra el pensamiento gomezdaviliano, que Rabier expone su tesis sobre una escritura de orden esotérico, metatextual: "En el uso metatextual o intertextual de los autores de su biblioteca, existe en Gómez Dávila el propósito evidente de esconder voluntariamente las fuentes de su pensamiento, o de recurrir a la alusión para dirigirse a una clase particular de lectores: los iniciados, los cultos, los que comparten con él principios y referencias. No se puede dejar de lado tampoco que el uso de la forma breve en Gómez Dávila obedece no solamente a un propósito estilístico, sino a esta misma estrategia esotérica en el sentido straussiano" (Rabier, 2013). 
de nuestras ideas en la estrechez de un sistema previamente determinado. La verdad, liberada de sus cualidades abstractas, es entendida, en cambio, como la relación de lo eterno y lo sensible, como presencia atemporal encarnada y que se nos regala en la experiencia concreta de la realidad:

Verdad no es aprehensión de objetos, ni contemplación de ideas, ni coherencia entre principios, sino posesión de un universal concreto. Verdad es el acto que alcanza, en la materia del objeto, la inexhausta plenitud del ser. La verdad es belleza, evidencia donde el objeto se consume en su inmovilidad de esencia (2002, p. 105).

Es a partir de esta inversión de la perspectiva tradicional de la inteligencia desde donde podemos comenzar a entender las condiciones particulares de la obra gomezdaviliana. En vista de que para él una vida consagrada a la inteligencia supone un movimiento constante, un dinamismo altivo y una soltura de las formas sistemáticas de escritura, entendemos entonces por qué este autor acusó a las exposiciones didácticas del pensamiento como representantes del quietismo de una inteligencia que se conforma únicamente con las conclusiones que le satisfacen. Ya las correctas palabras de Juan Fernando Mejía anticipaban, en su presentación de Gómez Dávila, en la Biblioteca de pensamiento colombiano del siglo XX, la caracterización del autor que se sigue a partir de las condiciones que nos plantea su obra:

La escritura de Gómez Dávila opone creatividad a productividad, honestidad a claridad, verdad a coherencia. El pensador no actúa ni como pedagogo ni como el moderno investigador: el pensador construye una relación con los actos del espíritu. De allí que el parámetro con que se miden sus verdades, sus aciertos o sus fracasos no puede ser un criterio objetivo externo en el que se encarne lo que sea oficialmente, pensamiento, saber o conocimiento. La norma está en la idea y la resistencia a la labor la pone el propio espíritu. El éxito o el fracaso lo indican en cada caso la fluidez y la elegancia de la frase, su capacidad para tocar el alma al tiempo que se remite al acto espiritual mismo(2007, p. 471).

Pareciera, pues, que a primera vista las particularidades de este pensador nos obligaran a renunciar a todo tipo de investigación académica en torno a su obra, o, por lo menos, a cualquier iniciativa que buscara organizar sus ideas dentro de los lineamientos de un sistema de pensamiento. En efecto, pese a que dichas iniciativas puedan llevarse a cabo, y pese a que en cierta medida corresponden al acto reflejo más natural frente a una obra fragmentaria y esparcida ${ }^{7}$, es evidente que cualquier 
tipo de sistematización estaría yendo en contra del espíritu de la obra misma y no tendría más remedio que dimitir o falsear al momento en que su pretensión de univocidad se viera truncada por el trasegar sorpresivo de un ejercicio de pensamiento que no teme incurrir en la contradicción.

Pese a lo anterior, no podemos perder de vista que el objetivo trazado al reconocer el valor del trabajo que nos lega Nicolás Gómez Dávila es encontrar las condiciones propias que la obra exige para su estudio, buscando con ello evitar los excesos que nos llevarían a ubicarla en un lugar tan reservado y distante hasta volverla inaprensible, o, bien, en un lugar tan cercano e informal hasta convertirla en anécdota. Más aún cuando sobre este punto bastaría escuchar la propia voz del bogotano al advertirnos que: "lo que aquí digo parecerá trivial a quien ignore todo a lo que aludo" (2005a, t. II, p. 335), o bien cuando afirma: "Mi verdad es la suma de lo que soy, no el simple resumen de lo que pienso" (2005a, t. I, p. 233) ${ }^{8}$.

Aunque el ejercicio de la inteligencia sea un ejercicio libre que busque substraerse de cualquier atadura o compromiso, y pese a que Gómez Dávila encarnó en su vida un esfuerzo altamente consagrado a esta convicción, no podemos pretender que su inteligencia se distinga entonces de todas las demás, y mucho menos cuando él no lo pensaba así. Indiferente a la originalidad de sus ideas, como en más de una ocasión nos dio a entender, Gómez Dávila nos muestra desde el comienzo de su obra que lo suyo no es un ejercicio hacia la originalidad, sino un trabajo de lucidez, un compromiso con esa cualidad díscola y valiente de la inteligencia que se lanza en una férrea reacción contra el facilismo, el embrutecimiento y el tedio que incuba un quehacer cotidiano irreflexivo. La intención de vivir la vida en la lucidez de la inteligencia podría entonces alternarse con la famosa motivación socrática a partir de la cual se entiende que una vida sin examen no merece la pena ser vivida9.

7 Recordemos, por ejemplo, que Gómez Dávila ha sido popularizado en Francia a través de selecciones temáticas de sus escolios escogidos y compilados por el editor Samuel Bruselle, en2003: Les horreurs de la démocratie - Scolies pour un texte implicite; y en 2005: Le Réactionnaire authentique, ambospublicadosporsu editorialAnatolia.

8 A lo anterior podríamos agregar también las siguientes afirmaciones: "La más simple verdad es tan compleja que ninguna fórmula la expresa, y requiere para expresarse el contexto global de una persona y de una vida" (Gómez Dávila, 2005a, t. I, p. 208) y "La verdad es persona" (p. 57).

$9 \quad$ Sobre este punto basta con referirnos a las primeras páginas de su libro Notas (primer libro que el autor publicó en vida),en las cuales, en un pasaje bastante personal e íntimo,nos confiesa el modo de vivir la inteligencia: "Ya que el orgullo me calla, intentaré entregarme a las delicias de una meditación que nada interrumpe. Inicio aquí un desfile monótono. 
Sin embargo, como cualquier otro intelectual, Nicolás Gómez Dávila vivió y pensó sobre la base activa de sus creencias y prejuicios. La única distinción posiblea este respecto, y quizás la más radical, esque a este autor bogotano no le importó en absoluto asumir sus creencias y prejuicios, en lugar de intentar fingir la apariencia de neutralidad que, para él, es casiun vicio en el que se incurre con más facilidad desde una perspectiva cientificista. Sobre este punto es inevitable no notar la consonancia con las valoraciones que de Joseph de Maistre realizaba Emil Cioran, cuando de este último decía:

Elevando el menor problema a la altura de la paradoja y a la dignidad del escándalo, manejando el anatema con una crueldad teñida de fervor, edificó una obra llena de excesos, un sistema que continúa seduciéndonos y exasperándonos. La magnitud y la elocuencia de sus cóleras, la vehemencia con que se entregó al servicio de causas indefendibles, su obstinación en legitimar más de una injusticia, su predilección por la expresión mortífera, definen a este pensador inmoderado que, no rebajándose a persuadir al enemigo, lo aniquila de entrada mediante el adjetivo. Sus convicciones poseen una apariencia de gran firmeza: a la tentación del escepticismo supo responder con la arrogancia de sus prejuicios, con la violencia dogmática de sus desprecios (1992, p. 12).

De esta forma aparece ante nosotros cuál ha de ser, según nuestra argumentación, el criterio que debería comenzar a orientar las investigaciones futuras que quieran dar cuenta de la profundidad intelectual de la obra de Gómez Dávila: buscar dilucidar de la forma más completa posible la basede las convicciones y creencias sobre las cuales este autor entabla su diálogo con las obras más representativas de la tradición occidental; presentarsus ideas a partir de la imagen del hombre que las piensa, pero reconstruir dicha imagen en la investigación de las fuentes históricas -que por sus escolios- sabemos que nutren sus convicciones. Esta conclusión se sustenta en la idea de que solo a través de rastrear el fondo intelectual de sus creencias, la datación

Sin presumir una importancia de que carecen estas notas, las escribo con una sencillez desinteresada, similar a nuestra actitud ante las imágenes que preceden al sueño. Las proclamo de nula importancia y, por eso, son notas, glosas, escolios; es decir, la expresión verbal más discreta y más vecina al silencio”.Además, más adelante concluye: “¿cómo vivir entregado a la sola tarea de vivir?, ¿cómo transitar por mis días, la frente inclinada sobre el instante, animal que pace, olvidado del cercano invierno y de la pura luz que lo circunda? Anhelo que estas notas, pruebas tangibles de mi desistimiento, de mi dimisión, salven de mi naufragio mi última razón de vivir. Imposible me es vivir sin lucidez, imposible renunciar a la plena conciencia de mi vida" (2003, pp. 49-50). 
de las ideas que alimentan sus prejuicios; sólo a través de establecer las líneas de pensamiento de las que él se siente un atemporal representante, es que vamos a comprender cabalmente la voz tan singular de este pensador que se vio a sí mismo como un amanuense de siglos.

Si nuestras reflexiones no van más allá de los propios textos de Nicolás Gómez Dávila, y no comienzan a situarlos en el diálogo intelectual desde el cual se forjaron, jamás podremos superar la dificultad que su "escritura esotérica" nos impone,y, lo que es peor, jamás podremos responder a su silencioso llamado.Sin una investigación extensa y a tiempo completo que remede en cierta medida la consagración total al estudio que significó buena parte de la vida de este colombiano, los cada vez más célebres escolios de este autor seguirán siendo para nosotros voces tan singulares como anecdóticas, y no la voz inconfundible y pura de quien dedicó su vida y su inteligencia a trazar estelas conminatorias entre escombros.

\section{REFERENCIAS}

AA.VV. (1988) "Homenaje a Nicolás Gómez Dávila”. Revista del Colegio Mayor de Nuestra Señora del Rosario, LXXXI, (542), abril-junio. Comprende escritos de Alberto Zalamea: Homenaje a Nicolás Gómez Dávila, p. 7; Francisco Pizano de Brigard, Semblanza de un colombiano universal-Las claves de Gómez Dávila, pp. 9-20; Hernando Téllez, La Obra de Nicolás Gómez Dávila, una dura punta de diamante, pp. 20-21. Álvaro Mutis, Donde se Vaticina el Destino de un Libro Inmenso, pp. 23-25; GerdKlaus Kaltenbrunner, Un pagano que cree en Cristo. El antimodernista colombiano Nicolás Gómez Dávila en alemán, pp. 31-33; Adolfo Castañón, Retratos de un pastor de libélulas: Nicolás Gómez Dávila, pp. 34-37; Nicolás Gómez Dávila, Antología: Notas pp. 38-44; Textos, pp. 45b-55; “Escolios inéditos”, pp.56-58; “De iure”, pp. 59-85.

Abad Torres, A. (2008). Pensar lo implícito: en torno a Gómez Dávila. Pereira: Centro de Recursos informáticos y Educativos-CRIE.

Brussell, S. (2003). Les horreurs de la démocratie - Scolies pour un texte implicite - Suivi de Un ange captif du temps par Franco Volpi. Anatolia Éditions du Rocher.

Brussell, S. (2005). Le Réactionnaire authentique. Choix de Samuel Brussell, 
préface de Martin Mosebach. Traduit de l'espagnol par Michel Bibard, Anatolia-Éditions du Rocher. ISBN: 2-268-05300-8.

Cioran, E. (1992). Ejercicios de admiración y otros textos. Barcelona: Tusquets.

Gómez Dávila, N. (2002). Textos I (2. a ed.). Bogotá: Villegas Editores.

Gómez Dávila, N. (2003). Notas(2. ed.). Bogotá: Villegas Editores.

Gómez Dávila, N. (2005a). Escolios a un texto implícito. Tomos I y II (2. ${ }^{a}$ ed.). Bogotá: Villegas Editores.

Gómez Dávila, N. (2005b). Nuevos escolios a un texto implícito. Tomo I y II (2. ed.). Bogotá: Villegas Editores.

Gómez Dávila, N. (2005c). Sucesivos escolios a un texto implícito (2. ${ }^{\text {a ed.). }}$ Bogotá: Villegas Editores.

Hoyos Vásquez, G. (1999). 'Medio siglo de filosofía en Colombia. Reflexiones de un participante". Revista de Estudios Sociales (3): 43-58. ISSN (versión en línea): 1900-5180.

Hoyos Vásquez, G. (2008). "Don Nicolás Gómez Dávila y reaccionario auténtico". ARBOR. Ciencia, Pensamiento y Cultura. CLXXXIV (734): 1085-1100, noviembre-diciembre.

Jaramillo Uribe, J. (1964). El pensamiento colombiano en el siglo XIX. Bogotá: Temis.

Laserna, M. (2001). "Nicolás Gómez Dávila, el hombre”, en: Gómez Dávila, N.: Escolios a un texto implícito (selección). Bogotá: Villegas.

Mejía Mosquera, J.F. (2007). Nicolás Gómez Dávila (1913-1994). En: Pensamiento colombiano del siglo XX. Tomo I. Castro-Gómez; FlórezMalagón, A; Hoyos Vásquez, G y Millán de Benavides, C. (Eds.). Bogotá: Editorial Pontifica Universidad Javeriana/Instituto PENSAR.

Oviedo, J. M. (1991). Breve historia del ensayo hispanoamericano. Madrid: Alianza.

Rabier, M. (2013). "Biblioteca gomezdaviliana: Las fuentes del pensamiento de Nicolás Gómez Dávila”. Rev. Interam. Bibliot. Medellín (Colombia), 36(3): 235-248, septiembre-diciembre. 
Rabier, M. (2014). "Nicolás Gómez Dávila y las paradojas del conservadurismo". La Razón Histórica, (26): 226-243.

Serrano Ruiz-Calderón, J. M. (2011) . "La libertad en la obra de Nicolás Gómez Dávila”. Revista Foro. Nueva Época. (14).

Volpi, F. (2005). Nicolás Gómez Dávila. El solitario de Dios. Bogotá: Villegas Editores. 